Mappemonde

Revue trimestrielle sur l'image géographique et les formes du territoire

$126 \mid 2019$

Varia

\title{
Le modèle économique du centre commercial chinois en Europe
}

The economic model of Chinese shopping centres in Europe

El modelo económico del centro comercial chino en Europa

\section{Li Minghuan}

Traducteur : Anne Bouhali

\section{OpenEdition}

\section{Journals}

Édition électronique

URL : http://journals.openedition.org/mappemonde/1245

DOI : $10.4000 /$ mappemonde. 1245

ISSN : 1769-7298

\section{Éditeur}

UMR ESPACE

\section{Référence électronique}

Li Minghuan, «Le modèle économique du centre commercial chinois en Europe », Mappemonde [En ligne], 126 | 2019, mis en ligne le 01 avril 2019, consulté le 11 novembre 2019. URL : http:// journals.openedition.org/mappemonde/1245;DOI : 10.4000/mappemonde.1245

Ce document a été généré automatiquement le 11 novembre 2019.

\section{(c) (i) (2)(2)}

La revue Mappemonde est mise à disposition selon les termes de la Licence Creative Commons Attribution - Pas d'Utilisation Commerciale - Partage dans les Mêmes Conditions 4.0 International. 


\title{
Le modèle économique du centre commercial chinois en Europe
}

\author{
The economic model of Chinese shopping centres in Europe \\ El modelo económico del centro comercial chino en Europa
}

Li Minghuan

Traduction : Anne Bouhali

Texte original : "Ouzhou huaren shangcheng jingji yanjiou” ("A study on Chinese malls economy in Europe"). Revue Shijie Minzu (« Population du monde ») 2013-3, p. 53-60.

Li Minghuan est professeure à l'Institut d'études des populations à l'Université de Xiamen en Chine et consultante au Bureau des affaires chinoises à l'étranger du Conseil des affaires d'État chinois. Sociologue, elle a publié plus de soixante articles dans des revues en anglais et en chinois sur la situation des migrants chinois en Europe et sur la politique des migrations internationales. Elle a également publié deux livres en anglais sur ces sujets. Le premier, tiré de sa recherche doctorale, est intitulé "We Need Two Worlds: Chinese Immigrant Associations in a Western Society, Amsterdam University Press, 1999. Le deuxième, "Seeing transnationally: how Chinese migrants make their dreams come true" (Leuven University Press, 2013), porte sur les réseaux transnationaux des entrepreneurs chinois en Europe. Cet article est tiré du projet « Ouzhou huaren yanjou » (« Recherche sur les Chinois d'outre-mer en Europe ») sponsorisé par le Financement National de Science Sociale du gouvernement chinois (11BMZ038). Il s'agit d'un article pionnier rédigé par la spécialiste chinoise de ces questions qui offre un point de vue sensiblement différent de celui communément porté par des travaux menés depuis l'Europe. 


\section{Introduction}

Depuis le début du XXI siècle, du fait de la croissance économique très rapide de la Chine, les Chinois d'outre-mer en Europe ont vu leur structure démographique et leur pouvoir économique profondément transformés. Ces transformations se manifestent particulièrement à travers le centre commercial chinois ${ }^{1}$, un modèle économique en forte progression sur le continent européen. Ce modèle est venu remplacer les niches économiques traditionnelles que sont, par exemple, les restaurants chinois, les ateliers textiles ou les petits commerces de proximité, devenant un nouveau modèle économique pour les communautés chinoises d'Europe en raison de son échelle et de son importance.

2 Ce nouveau marché chinois diffère du traditionnel "Chinatown ». Ces Chinatowns sont généralement situés dans des quartiers où se concentrent les résidents chinois à l'étranger. Bien que ces quartiers exotiques puissent attirer des habitants issus de la société d'accueil et des touristes, leur fonction principale consiste à fournir des services aux populations chinoises résidant à l'étranger. A contrario, les nouveaux marchés chinois (dont il est question dans ce papier) rassemblent des centaines voire des milliers de boutiques proposant des marchandises fabriquées en Chine. Leur modèle économique repose sur la vente en gros. Ces marchés sont ainsi devenus des plaques tournantes commerciales majeures pour les entrepreneurs issus de la diaspora chinoise, ainsi que des carrefours pour les circulations de produits chinois en Europe. En ce sens, les marchés chinois sont des lieux de rencontres directes entre les Chinois d'outremer et la société d'accueil, devenant ainsi l'objet de l'attention des hommes politiques, des médias et des citoyens originaires des pays de réception. Que les propriétaires de ces boutiques l'acceptent ou non, le marché chinois est devenu dans une certaine mesure le mode de représentation des produits made in China et de l'image de la Chine à l'étranger. Pour toutes ces raisons, le marché chinois est non seulement un phénomène économique, mais aussi un objet d'étude essentiel ayant une importance théorique et pratique.

3 Cet article s'appuie sur une analyse exhaustive de la littérature et sur des entretiens approfondis avec des entrepreneurs, réalisés à l'occasion de quatre enquêtes de terrain qui ont se sont déroulées entre 2011 et 2012 en France, en Italie, en Espagne, en Hongrie, en Russie et en Roumanie. En plus d'observations menées dans des centres commerciaux et des entretiens avec des entrepreneurs, j'ai considérablement enrichi ma compréhension de la question en discutant avec des universitaires et des responsables politiques locaux.

\section{Processus de développement de centres commerciaux chinois en Europe}

4 Les communautés chinoises d'outre-mer en Europe se caractérisent par une proportion importante de nouveaux migrants. D'après les statistiques de la Fédération européenne des associations chinoises (ouzhou huaqiao shetuan lianhehui), la diaspora chinoise présente en Europe ne comptait que 10000 ressortissants en 1950. Ce chiffre est passé à 50000 ressortissants dans les années 1960. Dans les années 1970, alors que les pays européens, dont la France, acceptaient un grand nombre de «boat people» ayant fui la 
guerre au Vietnam, le nombre de ressortissants a considérablement augmenté. Dès lors, de nouveaux migrants en provenance de Chine continentale n'ont cessé d'arriver en Europe. D'après le rapport annuel de l'Association européenne des Chinois d'outre-mer (ouzhou huaqiao huaren lianhe hui), le nombre total de membres de la diaspora chinoise en Europe a atteint 250000 personnes en 2008. Aujourd'hui, les migrants chinois sont présents dans toute l'Europe. Ils ont créé plus de 800 associations, plus de 300 écoles d'apprentissage de la langue chinoise et ont publié une centaine de journaux en chinois. Dans une certaine mesure, la diaspora chinoise d'Europe, composée principalement de migrants de première génération ayant quitté la Chine après 1978, a suscité beaucoup d'attention en Europe ${ }^{2}$.

5 Jusqu'à la fin des années 1980, les activités économiques des migrants chinois installés en Europe s'inscrivaient surtout dans l'économie de service, comme la restauration, la confection, la maroquinerie et la vente au détail. Sans compter des cas exceptionnels tels que le supermarché "Tang Frères », qui est devenu une entreprise importante au niveau national, la plupart des stratégies économiques chinoises se limitent à des marchés de niche. Dans les pays d'Europe orientale, le nombre de Chinois d'outre-mer est très faible; par conséquent, les activités économiques dont ils sont à l'origine s'écartent rarement du créneau que constitue le commerce ethnique.

6 Au tout début des années 1990, après la dissolution de l'URSS, la structure socioéconomique des pays d'Europe de l'Est a créé des occasions uniques pour les nouveaux migrants chinois. Une telle opportunité historique a coïncidé avec la croissance économique rapide de la Chine, conséquence de la créativité du peuple chinois ordinaire qui a émergé suite à la réforme économique de 1978. Le modèle économique des communautés chinoises d'Europe a donc changé. Le commerce international est ainsi devenu un nouveau modèle dominant qui s'est diffusé de l'Europe de l'Est vers l'Europe de l'Ouest et du Nord. Grâce à la productivité chinoise, les Chinois d'outre-mer ont créé d'énormes centres commerciaux multifonctionnels, associant fonctions d'entreposage, vente en gros et au détail. C'est ainsi qu'a émergé un nouveau modèle économique caractérisé par de vastes centres commerciaux, gérés par des migrants chinois nouvellement arrivés en Europe, et qui proposent des produits fabriqués en Chine.

7 Depuis leur naissance dans les années 1990, les centres commerciaux chinois situés dans les villes européennes ont ainsi connu une évolution en trois temps.

8 Les années 1990 ont été marquées par l'émergence des centres commerciaux chinois. À cette époque, de nombreux migrants chinois nouvellement arrivés introduisent les produits chinois à bas prix dans les marchés européens à travers différents canaux. Ces commerçants itinérants présentent des profils très variés : certains ont voyagé en Europe de l'Est et considèrent leur activité comme un moment de formation (liantan); d'autres sont devenus colporteurs (tilan jiaomai) dans le sud de l'Europe; d'autres enfin font la navette entre la Chine et la Russie pour vendre des marchandises déstockées. Les produits bon marché et inédits fabriqués en Chine par des entreprises locales privées ont alors connu un grand succès en Europe, en particulier auprès des classes moyennes et populaires. Quelques-uns des commerçants qui ont rejoint les premiers le marché et qui comptent parmi les plus prospères ont rapidement ouvert leur propre magasin de gros. Sous l'effet des «économies d'agglomération ", plusieurs marchés de gros de tailles différentes se sont développés dans les villes européennes. Parmi les plus fameux dans les années 1990, on trouve le «Four Tigers' Market» de Budapest, le « 
Market » et le «M Market» de la Piazza Vittorio à Rome, le «Centro Mourario» à Lisbonne, ou encore le quartier Sedaine-Popincourt à Paris, ainsi que le marché « Cherkízovsky rýnok» de Moscou. Tous ces marchés ont en commun le fait d'être concentrés dans l'espace, d'accueillir d'énormes flux de clients et de générer des bénéfices élevés grâce à de faibles coûts de production. Ils sont fondés sur le principe économique de la rapidité de l'accumulation primitive du capital. D'après un entrepreneur [chinois] rencontré en Italie, devenu riche de façon miraculeuse, «Pendant cette période, pas un seul propriétaire de magasin n'a connu la perte de profit... À l'époque, il était normal de vendre 400 à 500 conteneurs de produits par an ou 10 conteneurs par jour. » De nombreux entrepreneurs chinois parmi les plus riches aujourd'hui ont ainsi accumulé un capital initial durant cette période.

9 De 1990 à 2007, l'économie des migrants chinois est entrée dans une deuxième phase, celle de la croissance rapide et de la multiplication des marchés. Malgré la crise financière de 1998 qui a touché l'Europe, le marché chinois a résisté et a continué de croître, et ce en dépit du déclin mondial. Cette croissance continue a donné une forte impulsion aux entrepreneurs chinois qui dépendaient du marché chinois. En août 2007, le quotidien chinois en France "Europe Times » a ainsi publié un article faisant état de cette tendance : «De la Suède et de l'Angleterre à l'Espagne et l'Italie, toute l'Europe assiste à la multiplication des centres commerciaux chinois ». En France, dans la ville d'Aubervilliers, le CIFA ${ }^{3}$ 'est ainsi agrandi. Avec ses $40000 \mathrm{~m}^{2}$ et plus de 300 boutiques, le centre est devenu le plus grand centre de gros chinois d'Europe. En Allemagne, les marchés chinois se sont déployés dans tout le pays : de Leipzig et Düsseldorf en passant par Hambourg et Munich, plus d'une vingtaine de magasins et de marchés spécialisés dans la vente en gros ont ouvert. À Berlin et Francfort, on peut même trouver dans une même ville plusieurs marchés de gros chinois. En Italie, au Prato, un centre commercial chinois accueille plus d'une centaine de magasins spécialisés dans la vente de bijoux et de prêt-à-porter en gros. Il s'agit d'ailleurs du plus grand centre commercial jamais possédé par un ressortissant chinois. À Kalmar, en Suède, la construction d'un centre commercial chinois a débuté en février 2013. Enfin, le centre commercial Marco Polo, avec ses $150000 \mathrm{~m}^{2}$ commerciaux, est le plus grand centre commercial d'Italie. Les investisseurs ont d'ailleurs décidé d'en organiser la cérémonie d'inauguration à Pékin dans le Grand Hall du Peuple, en faisant ainsi l'exemple le plus représentatif des centres commerciaux chinois d'Europe.

10 Entretemps, l'économie chinoise a considérablement contribué au renouveau économique de l'Europe de l'Est. En Russie, les nouveaux migrants chinois représentent le principal pilier économique de plusieurs marchés. Ils jouent un rôle important dans les flux commerciaux entre la Chine et la Russie. En Serbie, le marché de gros « Numéro 70 » à Belgrade est extrêmement prospère, et propose des marchandises de toutes sortes, allant des aiguilles à coudre aux articles de décoration intérieure. Enfin, il convient de citer les exemples du marché de gros d'Ostrava en République tchèque et des marchés du Dragon rouge et du $7^{\mathrm{e}}$ kilomètre à Odessa, en Ukraine. Leur expansion se traduit également par la rénovation et la construction de nouveaux marchés.

11 Après la crise financière de 2008, la récession économique en Europe a précipité l'économie des migrants chinois dans une période de transition. Cette troisième phase est donc marquée par l'adaptation et la transition dans un contexte difficile. D'une part, le modèle économique des centres commerciaux chinois s'est amélioré au point que ces centres commerciaux accordent désormais une plus grande attention à 
l'environnement, à l'hygiène et à la propreté. Les entrepreneurs cherchent également à innover. D'autre part, la concentration spatiale des centres commerciaux a eu des retombées sociales négatives. Tous les problèmes engendrés par les activités des entrepreneurs chinois ont été évoqués dans les médias des pays d'accueil. Ils ont même parfois pu provoquer des troubles diplomatiques avec la Chine.

\section{Typologie des centres commerciaux chinois en Europe}

Tableau 1. Centres commerciaux chinois en Europe

\begin{tabular}{|c|c|c|c|c|}
\hline Pays & Ville & Marché & Type de construction & Investisseurs \\
\hline \multirow{3}{*}{ France } & Paris & Popincourt & $\begin{array}{l}\text { Concentration de } \\
\text { boutiques dans un } \\
\text { quartier de centre-ville }\end{array}$ & $\begin{array}{l}\text { Grossistes } \\
\text { indépendants }\end{array}$ \\
\hline & \multirow{2}{*}{ Aubervilliers } & CIFA & $\begin{array}{l}\text { Centre commercial } \\
\text { nouvellement/ } \\
\text { récemment construit }\end{array}$ & $\begin{array}{l}\text { Société } \quad \text { immobilière } \\
\text { française }\end{array}$ \\
\hline & & Fashion Centre & $\begin{array}{l}\text { Centre commercial } \\
\text { nouvellement/ } \\
\text { récemment construit }\end{array}$ & Commerçants chinois \\
\hline Italie & Rome & Piazza Vittorio & $\begin{array}{ll}\text { Concentration } & \text { de } \\
\text { boutiques dans un } \\
\text { quartier }\end{array}$ & Commerçants chinois \\
\hline Espagne & Madrid & Fuenlabrada & $\begin{array}{ll}\text { Utilisation } & \text { d'une } \\
\text { ancienne } & \text { friche } \\
\text { industrielle } & \end{array}$ & Commerçants chinois \\
\hline \multirow{5}{*}{ Russie } & \multirow{5}{*}{ Moscou } & $\begin{array}{l}\text { Cherkízovsky } \\
\text { rýnok }\end{array}$ & Marché à ciel ouvert & Propriétaire local \\
\hline & & Luniki Stadium & Marché de plein air & \\
\hline & & $\begin{array}{l}\text { Central Dynamo } \\
\text { Stadium }\end{array}$ & Marché de plein air & \\
\hline & & Greenwood Park & $\begin{array}{l}\text { Centre commercial } \\
\text { nouvellement/ } \\
\text { récemment construit }\end{array}$ & $\begin{array}{l}\text { Joint-venture entre une } \\
\text { entreprise chinoise (Groupe } \\
\text { Hengtong) et la mairie de } \\
\text { Moscou }\end{array}$ \\
\hline & & $\begin{array}{l}\text { Yiwu } \\
\text { international } \\
\text { trading center }\end{array}$ & $\begin{array}{l}\text { Centre commercial } \\
\text { nouvellement/ } \\
\text { récemment construit }\end{array}$ & $\begin{array}{l}\text { Joint-venture entre des } \\
\text { groupes privés chinois et } \\
\text { une entreprise russe }\end{array}$ \\
\hline & Bucarest & Niro market & Marché à ciel ouvert & Propriétaire local \\
\hline
\end{tabular}

Roumanie 


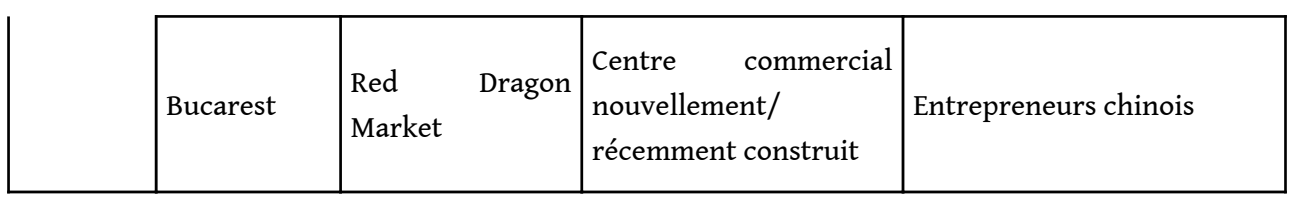

12 Les centres commerciaux les plus importants d'Europe sont aujourd'hui les suivants: Aubervilliers, Popincourt dans le $11^{\mathrm{e}}$ arrondissement parisien, la Piazza Vittorio à Rome, le Fuelenbrada de Madrid, le "Wenzhou Center», le «Monori Center» et «Eurosquare » à Budapest, le "Red Dragon Market» en Roumanie et le "Monori Center » à Moscou. Les processus de développement des centres commerciaux chinois ne sont pas identiques entre Europe de l'Est et de l'Ouest du fait d'environnements économiques différents. Il est ainsi possible de distinguer trois catégories de marchés de gros chinois selon le type de construction.

Le premier type de marché est le marché à ciel ouvert. Ces marchés résultent souvent de la concentration spatiale d'entrepreneurs chinois. Ils sont principalement situés en Europe de l'Est, notamment en Russie. Au début des années 1990, à Moscou, quelques centaines de marchés de plein air sont ainsi apparus autour de stations de métros, et même autour de stades nationaux (le Luniki Stadium, ou le Central Dynamo Stadium), formant ainsi un vaste réseau commercial de vente de marchandises de qualité faible et moyenne. Ces marchés sont appréciés pour leur faible coût d'investissement et leur modèle commercial simple. Avant septembre 2008, les Chinois de Moscou appelaient le marché de Cherkízovsky rýnok «le marché de la grande fourmi », un marché qui s'est depuis étendu à Moscou pour atteindre plusieurs centaines d'hectares. Bien que ces marchés aient une apparence extrêmement simple et soient peu attrayants - certaines des soi-disant «boutiques" sont tout bonnement des conteneurs abandonnés -, les transactions quotidiennes réalisées dans ce type de marché peuvent atteindre jusqu'à 50 voire 60 millions de dollars, tandis que des dizaines de milliers de Chinois y vivent et y travaillent. Un autre exemple de ce type de marchés est le "Grand marché chinois » d'Iekaterinbourg, en Sibérie. D'une superficie de plus de $40000 \mathrm{~m}^{2}$, les produits textiles made in China représentent $95 \%$ des produits vendus localement. Sur les 60000 magasins, plus de $90 \%$ des propriétaires sont chinois. Leurs magasins sont faits de tôle, et les marchandises qui y sont vendues sont presque exclusivement fabriquées en chine.

14 Le deuxième type de centre commercial chinois se présente sous la forme d'une concentration de boutiques chinoises à l'intérieur d'un quartier. Bien qu'à Paris, le $13^{\mathrm{e}}$ arrondissement ait formé un marché principalement composé de Chinois d'outre-mer venus notamment des anciennes colonies françaises (Laos, Cambodge), mais aussi d'ailleurs en Asie du Sud-est (Indonésie), l'image du quartier est celle d'un Chinatown traditionnel, avec des magasins, des restaurants, des agences de voyage ou des salons de beauté appartenant à des Chinois. On retrouve ce type de quartier en Angleterre (Londres, Manchester), aux Pays-Bas (Rotterdam, Amsterdam) ou encore en Espagne (Madrid). Notre étude porte ici sur un autre type d'économie. Alors que les premiers modèles de marché étaient centrés sur le commerce de détail, les centres commerciaux chinois - concentrés principalement dans un seul quartier - reposent désormais sur le commerce de gros. Les marchandises qui y sont vendues sont très peu diversifiées (vêtements ou articles de maroquinerie), à destination surtout des détaillants. Par conséquent, le marché chinois en Europe est avant tout un commerce de gros, avec une spécialisation dans le textile, l'électronique et le jouet pour enfant. Les flux de clients, 
de véhicules, de marchandises et de capitaux impliqués dans le commerce de gros sont beaucoup plus importants que ceux que génèrent les commerçants des Chinatowns traditionnels. Dans cette optique, les deux types de quartiers ne sont donc pas comparables. Par exemple, à Madrid, le marché chinois de Fuenlabrada concentre plus de 300 boutiques de gros et 3000 boutiques de détail. À Paris, dans le $11^{\mathrm{e}}$ arrondissement, la rue Popincourt concentre 40 boutiques de vente en gros là où l'on trouvait auparavant une cinquantaine de magasins relevant de secteurs très divers. En Italie, dans le quartier situé à côté de la Piazza Vittorio, plus de 500 magasins de gros appartiennent à des entrepreneurs chinois et sont répartis le long des différentes avenues qui traversent le quartier.

Enfin, un troisième type de centre commercial repose sur la rénovation d'anciens entrepôts ou d'anciennes usines, ou bien sur la construction d'un nouveau bâtiment à la place d'anciennes friches industrielles, et qui sont ensuite divisés ou loués à des grossistes. Aubervilliers en est un parfait exemple. Avant les années 1990, ce quartier était une friche industrielle; aujourd'hui, il est devenu une plaque tournante commerciale et compte près d'un millier de magasins de gros. Le marché « Red Dragon » en Roumanie et le "Greenwood International Trading Center » à Moscou illustrent également cette tendance.

\section{Qui sont les investisseurs de ces marchés chinois en Europe?}

16 Modèle économique sans précédent en Europe géré par des entrepreneurs immigrés, le centre commercial chinois est un pilier économique pour les migrants chinois européens, une plateforme de circulation commerciale entre la Chine et l'Europe, ainsi que le maillon d'un réseau commercial mondial de produits fabriqués en Chine. En somme, les acteurs qui investissent dans ces centres commerciaux chinois en Europe sont de cinq types: des entrepreneurs non chinois issus de la société locale, des entrepreneurs-migrants chinois qui vivent en Europe, des entrepreneurs issus d'autres minorités, des entrepreneurs de Chine et des entreprises d'État chinoises. Ces investisseurs ont formé plusieurs types de partenariat : parfois ils travaillent ensemble, parfois seuls ; conflits et collaborations coexistent. Ces deux ou trois dernières années, une nouvelle tendance a émergé: certains riches entrepreneurs et entreprises chinoises en Chine ont fini par considérer l'immobilier comme un investissement rentable, et y dépensent donc beaucoup d'argent. L'exemple le plus frappant est celui du «Fashion center» à Aubervilliers, situé en banlieue nord de Paris. Dans les années 1970, Aubervilliers a connu un processus de désindustrialisation, de nombreuses entreprises quittant la ville pour s'installer à l'étranger. Un groupe d'entrepreneurs juifs y a vu une opportunité d'affaires : ils ont acheté les usines abandonnées, rénové les anciens entrepôts en salles d'exposition et les ont loués à des grossistes juifs. Dans les années 1990, certains entrepreneurs chinois ont été attirés par la présence de ce commerce de gros et y ont installé leurs propres magasins. Les grossistes chinois étant de plus en plus nombreux, les entrepreneurs juifs, principaux propriétaires immobiliers, en ont donc tiré bénéfice.

17 Le développement de la présence des grossistes chinois à Aubervilliers n'a cessé d'attirer l'attention des promoteurs immobiliers. En octobre 2006, des sociétés immobilières françaises ont investi des sommes considérables pour créer un centre de 
vente en gros «sur mesure » nommé «CIFA ». La première vague de la construction proposait $15000 \mathrm{~m}^{2}$ commerciaux, avec 95 magasins mesurant de 110 à $400 \mathrm{~m}^{2}$ chacun. La proposition du CIFA est née au moment de l'âge d'or du marché de gros d'Aubervilliers : avant même l'inauguration du centre, ses magasins étaient déjà tous vendus. Un an plus tard, l'entreprise proposait la deuxième phase du CIFA. En 2012, en pleine crise financière mondiale, huit entrepreneurs chinois collaboraient à un nouveau projet de construction pour un coût d'un milliard d'euros : celui du «Fashion Center ». D'une surface totale d'environ $5500 \mathrm{~m}^{2}$, il devrait accueillir plus de 300 magasins de gros, créer plus de 2800 emplois, pour des flux financiers estimés à 4,5 millions d'euros.

18 À Bucarest, de l'«Europa Center» au "Red Dragon", une partie de l'immobilier appartenait à des Roumains. Selon les dirigeants des communautés chinoises locales interrogés, les deux marchés chinois étaient détenus par la société Niro, une société locale appartenant à des entrepreneurs de nationalité roumaine. Afin d'accroître ses bénéfices, la société Niro n'a cessé de modifier sa stratégie de location, forçant même les grossistes chinois à louer de nouveaux magasins. Dans ces conditions, les grossistes chinois ont organisé en Roumanie le "Tangrenjie Realestate Group» (littéralement le "Chinatown ») et ont collaboré avec une société dont le siège se trouve en Turquie, et qui détenait des terrains à Bucarest. Ils ont investi environ 1 milliard d'euros et ont construit un marché de gros d'une surface de $250000 \mathrm{~m}^{2}$, capable d'accueillir plus de 200 magasins. Malheureusement, le centre ayant été créé au moment de la crise de la dette européenne, la conjoncture économique n'a pas été propice à l'essor des entreprises chinoises. Cette situation structurelle a été aggravée par les conflits internes entre les investisseurs de Tangrenjie, et les retombées économiques n'ont donc pas été optimales.

19 À Moscou, le plus grand projet est le "Greenwood Commercial Center », qui a été principalement financé par le groupe Hengtong. Situé au nord de la capitale russe et inauguré le 16 septembre 2012, le "Greenwood Commercial Center " s'étend sur une superficie de plus de 20 hectares. D'après la présentation du centre de commerce international de Greenwood, l'objectif de ce développement commercial était de construire une plateforme de collaboration économique efficace entre la Chine et la Russie. Cette plateforme suivrait ainsi le programme du gouvernement chinois visant à favoriser la marchéisation ${ }^{4}$ de son économie et à promouvoir des améliorations structurelles pour les entrepreneurs chinois à l'étranger. Le "Yiwu International Trading Center ", également à Moscou, est un projet similaire, réalisé grâce aux investissements de la société Shifeng, dont le siège se trouve au Zhejiang, en association avec la municipalité de Moscou et le groupe russe Tashir, pour un investissement total de 5 milliards de dollars. La ville de Moscou est l'investisseur principal du projet, à hauteur de $40 \%$ du capital, ce qui en fait d'ailleurs le projet le coûteux dans lequel la ville ait investi. Une fois construit, le centre pourra accueillir plus de 1900 magasins, tous spécialisés dans la vente de produits fabriqués en Chine ${ }^{5}$.

\section{Caractéristiques de l'économie des centres commerciaux chinois en Europe}

L'économie des migrants chinois installés en Europe présente quelques grandes caractéristiques: les magasins sont petits, les stocks sont importants, le niveau de 
compétence requis pour entrer dans les affaires est faible, et l'économie d'agglomération joue à plein. De la concentration spatiale résultent notoriété et popularité, ce qui attire une clientèle nombreuse. De plus, le marché chinois est aussi un forum qui permet aux entrepreneurs individuels d'avoir accès efficacement à l'information. De ce fait, les entreprises d'un même secteur peuvent facilement créer des liens et ainsi élargir leur base commerciale.

21 La plupart des pays d'Europe occidentale sont des États providence avec un régime de taxation élevée. Le marché des marques locales est déjà mature et manque de produits moyenne gamme ou bas de gamme. Le marché chinois repose principalement sur des produits bas de gamme importés de Chine. Son modèle de gestion est plus ou moins caractérisé par des pratiques informelles: certains commerçants comptent sur la relation de confiance et se fournissent chez le grossiste avant de le payer, d'autres grossistes prennent les commandes d'entreprises locales réputées.

Parmi les autres pratiques situées dans cette "zone grise» figurent les sousdéclarations (huise qinguan), la surcharge de travail, etc. Quelle que soit la stratégie employée, ces pratiques créent généralement une distorsion de la concurrence par rapport aux entrepreneurs locaux.

Dans une perspective favorable, le marché chinois se compose de produits bas et moyens de gamme qui complètent les produits haut de gamme déjà existants sur le marché local. Les clients des grossistes sont principalement composés d'autres entrepreneurs qui sont aussi des immigrants. En ce sens, les classes moyennes et inférieures de la société locale bénéficient directement du commerce de gros. En revanche, le marché chinois ne se distingue pas vraiment du modèle du marché en plein air. La plupart des magasins continuent de fonctionner sur le modèle des « ateliers familiaux » qui reposent sur la relation familiale et maximisent leurs profits grâce à des filières informelles.

24 L'économie informelle profite ainsi des zones d'ombre de l'économie formelle. Ces "échappatoires" tendent à permettre au marché de se soustraire au contrôle juridique. De fait, lorsque l'économie des pays européens fonctionne bien, les contrôles réglementaires et juridiques sont peu fréquents; cependant, en période de crise financière, les gouvernements européens ont tendance à renforcer leur contrôle afin d'augmenter les recettes. Après la crise financière de 2008, l'économie européenne a connu un déclin et des débats animés ont eu lieu dans un contexte de désaccords économiques et d'hostilités ethniques. Par conséquent, les centres commerciaux chinois sont également devenus une cible pour les critiques et les autorités de réglementation.

\section{Les problèmes sociaux et de sécurité des centres commerciaux chinois [non traduit]}

[Nous avons décidé de ne pas traduire cette section, car elle est moins pertinente par rapport à la problématique de ce dossier thématique.] 


\section{Perspectives des marchés de gros européens} les entrepreneurs locaux. En Asie du Sud-Est, la coopération entre les gouvernements chinois et locaux est bien connue. Dans mon enquête, j'ai remarqué que les entrepreneurs chinois installés en Europe ont tendance à suivre des chemins similaires à ceux de leurs homologues du Sud-Est asiatique. En Europe, la plupart des pays fonctionnent sur le système du multipartisme. L'alternance entre partis de droite et partis de gauche est fréquente, et les politiques migratoires sont très fluctuantes. Face à cette situation, certains entrepreneurs préfèrent inviter des responsables locaux à visiter la Chine et à y rencontrer les dirigeants chinois. Ce type de stratégie non seulement augmente leurs ressources symboliques, mais permet également de créer des liens étroits entre les dirigeants chinois et les responsables locaux européens. D'autres entrepreneurs ont engagé des fonctionnaires retraités comme consultants pour leur entreprise, ou les ont même parfois nommés PDG, afin de constituer des réseaux sociaux locaux pour leur entreprise.

31 Pour conclure, une juste répartition des parts de marché et la coexistence avec la société locale sont des conditions nécessaires pour que les entrepreneurs chinois en 
Europe puissent réaliser harmonieusement leurs plans de développement et d'intégration ${ }^{7}$. Le système de l'État Providence européen repose sur des impôts élevés. Si les entrepreneurs chinois tirent parti des systèmes locaux de protection sociale, ils doivent également être conscients de leur responsabilité sociale de créer de la richesse pour les sociétés et les populations locales ${ }^{8}$. Un groupe minoritaire qui crée des opportunités d'emploi et des revenus imposables pour sa société d'accueil gagnera nécessairement le respect et la reconnaissance des gouvernements locaux et des citoyens, ce qui permettra de faire naître une solidarité entre la société d'accueil et leur pays d'origine. Il est ainsi nécessaire que les grossistes chinois normalisent leurs pratiques commerciales dans le respect du droit commun, ce qui améliorera en même temps leur image.

\section{NOTES}

1. Le nom de ce type de marché et de ce type de quartier varie d'un pays à l'autre: «zone économique des Chinois d'outre-mer" (huaren shangmao cheng), "centre commercial Chine " (zhongguo shangcheng), "centre commercial Asie» (yazhou shangcheng). Dans cet article, nous avons opté pour l'expression « centre commercial chinois » (huaren shangcheng).

2. Au sujet de l'évolution et du développement des communautés chinoises d'outre-mer en Europe, voir Li Minghuan 2002, «Ouzhou Huaqiao Huaren Shi» (« Histoire des Chinois d'outre-mer en Europe »), Zhongguo huaqiao chuban she. Concernant le changement structurel des communautés chinoises depuis le début du XXI siècle, consulter Li Minghuan, 2009 "Ouzhou huaren shehui poxi » («Analyse des communautés chinoises d'outre-mer en Europe »), Shijie Minzu (2009: 5).

3. Centre international France-Asie.

4. Marchéisation : en finances, fait d'utiliser de plus en plus les marchés financiers, spécialement pour les banques. Source : Encyclopaedia Universalis.

5. Ces deux projets immobiliers ont été initiés avant la crise économique de 2008. Les deux centres commerciaux ont été construits durant la crise et ont été inaugurés au moment où la crise s'aggravait. Alors que le marché de gros existant était en déclin en raison de la crise, la proposition de plusieurs centaines de nouveaux magasins de gros semblait vouée à l'échec. Au moment de la rédaction de cet article, plusieurs showrooms n'étaient pas encore loués. De plus, ces deux centres commerciaux se ressemblent beaucoup en termes de design architectural et de modèle commercial. De ce fait, la concurrence est encore plus brutale. Il est important pour les investisseurs et les gestionnaires des centres commerciaux de trouver des solutions pour une concurrence plus saine.

6. Il est à noter que, dans certaines petites villes, le climat xénophobe est si répandu que les centres commerciaux chinois sont devenus objets de discrimination. Par exemple, en 2010 en Espagne, un groupe d'entrepreneurs chinois a proposé de construire un centre commercial de $900 \mathrm{~m}^{2}$ dans la ville d'Olivares. Le projet a rencontré l'opposition des résidents et des commerçants, surtout des petits entrepreneurs, de la ville. Sous la pression des citoyens, la municipalité a fini par adopter une nouvelle loi connue sous le nom de « loi PGOU », qui a interdit aux entrepreneurs chinois d'ouvrir de nouveaux magasins à Olivares, en particulier des épiceries et des bazars. 
7. Je remercie M. Dai Xiaozhang pour notre discussion qui a eu lieu en Italie.

8. Une partie des entrepreneurs chinois comprend l'importance d'un tel comportement pour contribuer à la société locale. Par exemple, à l'hiver 2012, une tempête de neige a causé la mort d'une centaine de personnes en Europe centrale. Les grossistes chinois de Bucarest ont alors réuni un fonds de secours à destination des sinistrés qu'ils ont fait parvenir à la zone la plus touchée par la tempête. À Budapest, l'Association des femmes (Huaqiao funu lianhehui) a fait des dons chaque hiver depuis cette catastrophe à destination des orphelins et des personnes âgées.

\section{RÉSUMÉS}

Depuis le $\mathrm{XXI}^{\mathrm{e}}$ siècle, les centres commerciaux chinois se sont rapidement développés dans les métropoles européennes. Que ce soit à Paris, Rome, Madrid ou Moscou, les centres commerciaux sont devenus une des modalités de la présence de la Chine à l'étranger, à travers la concentration spatiale de magasins. Ce papier retrace le contexte historique et social qui a contribué à la formation de centres commerciaux chinois en Europe. Il propose ensuite une typologie de ces centres commerciaux selon leurs caractéristiques. Enfin, il conclut par les défis et les problèmes sociaux auxquels sont confrontés ces centres commerciaux dans le contexte européen de l'aprèscrise économique de 2008.

Starting at the turn of the 21st century, Chinese shopping centres have rapidly developed in Europe's major cities. Be it Paris, Rome, Madrid or Moscow, these centres, through the spatial concentration of shops, have become one of the modalities of China's presence abroad. This article retraces the historical and social context that has contributed to the development of Chinese shopping centres in Europe. It then offers a typology of these centres according to their characterisitics. Finally, it outlines the social challenges and problems facing these centres in the European context during the aftermath of the 2008 economic crisis.

Los centros comerciales chinos se han desarrollado rápidamente en las metrópolis europeas desde inicios del siglo XXI. Su creciente concentración espacial en París, Roma, Madrid o Moscú visibilizan la presencia de China en el exterior. Este artículo propone una categorización de esta tipología comercial en Europa y explica el contexto histórico y social que ha favorecido su formación. Por otra parte, señala los desafíos y conflictos a los cuales se enfrenta este modelo de actividad en Europa después de la Gran Recesión de 2008.

\section{INDEX}

Thèmes : Les routes de la soie existent déjà. Routes transnationales et places marchandes du made in China entre Asie Afrique et Europe

Palabras claves : centros comerciales chinos, Chinos de ultramar en Europa, intercambio comercial China-Europa

Keywords : chinese malls, overseas Chinese in Europe, Chinese-European trade

Mots-clés : centres commerciaux chinois, Chinois d'outre-mer en Europe, commerce ChineEurope 